\title{
A Pelvic Mass Masquerading as High Stage Malignancy - An Interesting Presentation of Psuedo-Meigs Syndrome
}

\author{
Cathy Monteith, PhD ${ }^{1^{*}}$ (D), Tamara Kalisse ${ }^{1}$ (D), Robert A Farrell ${ }^{1}$ (D), Yulia Shahabuddin ${ }^{1}$ and Thomas \\ John D'Arcy, MD ${ }^{1,2,3}$
}

${ }^{1}$ Department of Gynaecology, St. James Hospital, Dublin, Ireland

${ }^{2}$ Coombe Women's and Infant's University Hospital, Dublin, Ireland

${ }^{3}$ Trinity College Dublin, Dublin, Ireland

*Corresponding author: Dr. Cathy Monteith, PhD, Department of Gynaecology, St. James Hospital, Dublin, Ireland, Tel: $+353-14103000$

\begin{abstract}
The lifetime risk of developing an ovarian malignancy is reported to be $1.4 \%[1,2]$. Invasive epithelial ovarian cancers have the highest prevalence in women between 60-64 years while germ cell or borderline tumours pre-dominate in women under 40 years [2]. We report the case of a 33-yearold nulliparous woman with a $7 \mathrm{~kg}$ leiomyoma, extensive endometriosis and several radiological features suggestive of a high stage ovarian malignancy. Meigs syndrome typically presents as a triad of ascites, pleural effusion and a benign ovarian fibroma [3]. Psuedo-meigs syndrome differs by presentation with ascites, pleural effusion and alternative benign tumours of the ovary such as mucinous cystadenomas, teratomas, struma ovarii and uterine leiomyomas [4]. However, in this reported case we demonstrate normal ovarian appearance and histology.
\end{abstract}

\section{Keywords}

Endometriosis, Ovarian cancer, Neoplasms, Leiomyoma, Fibroid, Meigs syndrome

\section{Introduction}

The lifetime risk of developing an ovarian malignancy is reported to be $1.4 \%[1,2]$. Invasive epithelial ovarian cancers have the highest prevalence in women between 60-64 years while germ cell or borderline tumours pre-dominate in women under 40 years [2]. Meigs syndrome, first described by Meigs typically presents as a triad of ascites, pleural effusion and a benign ovarian fibroma [3]. A similar presentation is described in pseudo
Meigs syndrome which differs in presentation with ascites, pleural effusion and alternative benign tumours of the ovary such as mucinous cystadenomas, teratomas, struma ovarii or uterine leiomyomas [4]. Uterine leiomyomas are the most common benign gynaecological neoplasm with a reported lifetime risk of 30\% [5].

\section{Case Description}

We present a 33-year-old nulliparous woman with a previous history of midline laparotomy and right oophorectomy in another institution for a ruptured Dermoid cyst. She presented to our unit with a two year history of progressive painless abdominal distension, weight loss, bilateral pitting oedema and cyclical bleeding from the umbilicus. She delayed seeking medical attention as she was trying to conceive. On clinical examination she was cachectic, had palpable cervical lymph nodes and auscultation of the lungs demonstrated reduced air entry. Palpation of abdomen revealed an irregular mass arising from the right iliac fossa, non-mobile, extending to the right upper quadrant and a second additional separate cystic mass palpable in left abdominal wall. Laboratory data were normal with the exception of elevated Ca125 of 233.

A computed tomography (CT) revealed a large left pleural effusion, a $26 \times 16 \times 31 \mathrm{~cm}$ solid heterogenous mass with low attenuation areas suggestive of necrosis and overall appearance suggestive of ovarian malignan- 
cy. In addition, there were multiple calcified lesions especially in the right upper quadrant abutting the liver representing possible metastatic deposits. There was large volume ascites and a $19 \times 4 \mathrm{~cm}$ fluid collection in the left anterior abdominal wall.

A pleuritic drain was inserted and yielded serous fluid with cytology negative for malignancy and pelvic biopsy showed no evidence of necrosis, atypia or increased mitoses and was in keeping with a smooth muscle lesion likely representing a benign leiomyoma. With the clinical and radiological suspicions, the gynaecology multidisciplinary meeting recommended progressing surgery with pre-operative insertion of right ureteric stent, interval reduction in pleural effusion, coagulation specialist opinion regarding insertion of a prophylactic IVC filter (advised prophylactic heparin only) and prophylactic uterine artery embolization which was performed six days pre-operatively.

The team proceeded to a total abdominal hysterectomy due to grossly distorted uterus inseparable from 7 kg mass, left salpingectomy, biopsy and conservation of left ovary due to normal gross appearance, appendectomy, excision of presumed abdominal wall seroma, infra-colic omentectomy, refashioning of abdominal wall, drainage of $2.6 \mathrm{~L}$ ascites, estimated blood loss $1000 \mathrm{ml}$.

Intra-operative ascitic fluid was negative on cytology for malignancy. Histopathology reported a $28 \mathrm{~cm}$ leiomyoma with hyalinization, ischaemic necrosis secondary to embolization and extensive adenomyosis. There was extensive endometriosis of the anterior abdominal wall, umbilicus, omentum and seroma with endometriotic cyst formation. The perihepatic calcifications were blood clot associated with aggregated macrophages, acute inflammatory cells and fragments of mesothelium. Left ovarian biopsy demonstrated normal ovarian stroma with no evidence of endometriosis and similarly normal assessment of the appendix and left fallopian tube.

The early post-operative course was complicated with a third order pulmonary embolism and transfusion of four units red cells to correct anaemia. The ureteric stent was removed at three months post-operatively. At the six month post-operative review the patient reported an uncomplicated physical recovery with appropriate weight gain, healthy appearance, laboratory confirmation of preserved ovarian function with anti-mullerian hormone of 24 and satisfactory cosmetic result of abdomen. She was discharged at that visit to her general practitioner and a reproductive counsellor.

\section{Conclusion}

Ovarian cancer remains one of the most frequent causes of death at $47 \%$ of all deaths from cancers of the female genital tract [2]. Women with ovarian cancer often report only vague symptoms of bloating, abdominal distension and as a result frequently present at a late stage (Stage III or IV) of disease in two thirds of cases with associated ascites or pleural effusions [2].

The aetiology of the observed ascites and pleural effusion in Meigs or Psuedo Meigs syndrome is unclear. The suggested mechanisms have included hypo albuminemia, peritoneal irritation, lymphatic obstruction, release of toxins or inflammatory mediators [6]. There is limited literature surrounding fertility conservation in the setting of Meigs syndrome including those occurring in paediatric cases [7]. The attainment of a presurgical diagnosis of Meigs or Psuedo Meigs syndrome remains challenging especially in the setting of an elevated $\mathrm{Ca}$ 125.

Ca 125 is a glycoprotein which was first associated and measured as a marker for established ovarian cancer in the early 1980s [8]. While it does not offer a role in screening for ovarian cancer, a serum Ca 125 level is a vital component of the risk of malignancy index (RMI) which is used to triage cases of suspected malignancy. An increased serum Ca 125 has shown ethic variation with higher levels in Caucasian women when compared to Black or Asian women. The following conditions have also demonstrated increased serum Ca 125 levels: Pelvic inflammatory disease, fibroids, cyst accident, endometriosis, tuberculosis, cirrhosis, ascites, hepatitis, pancreatitis and pleuritis. Elevated levels have also been demonstrated in primary breast, pancreas, lung

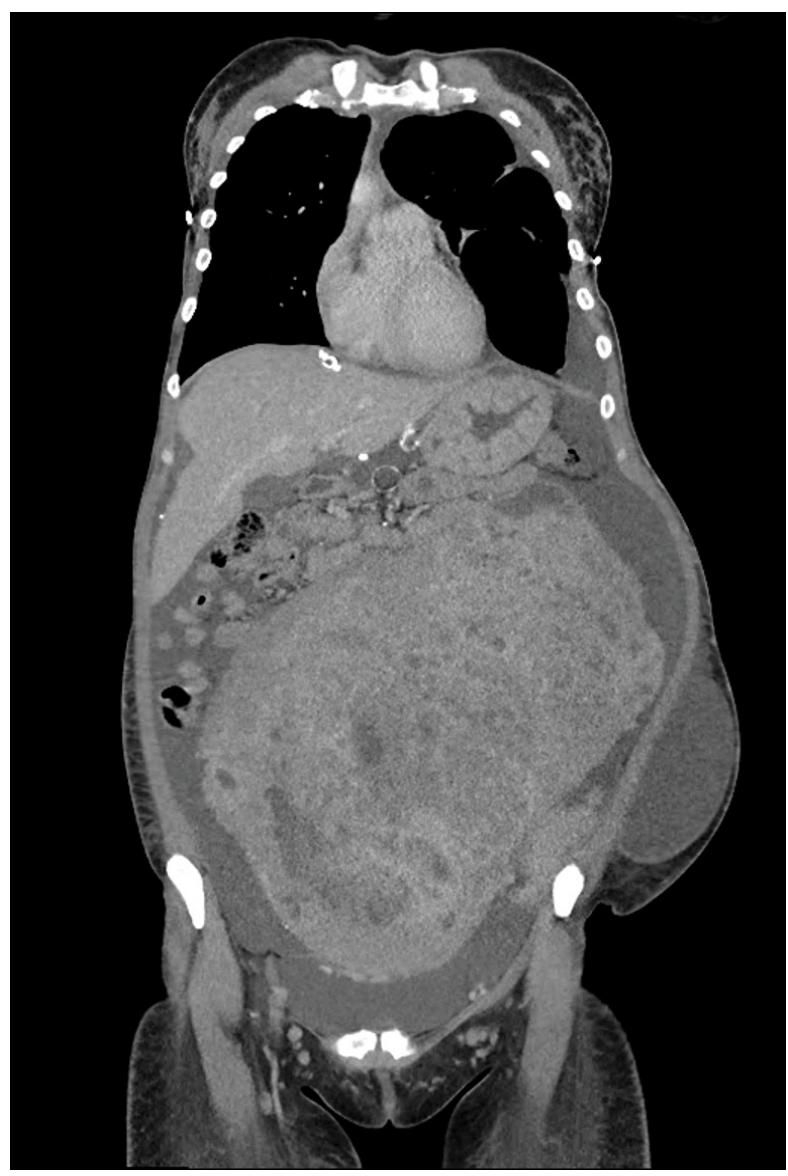

Figure 1: Pre-operative CT imaging of a patient with Psuedo-Meigs Syndrome. 


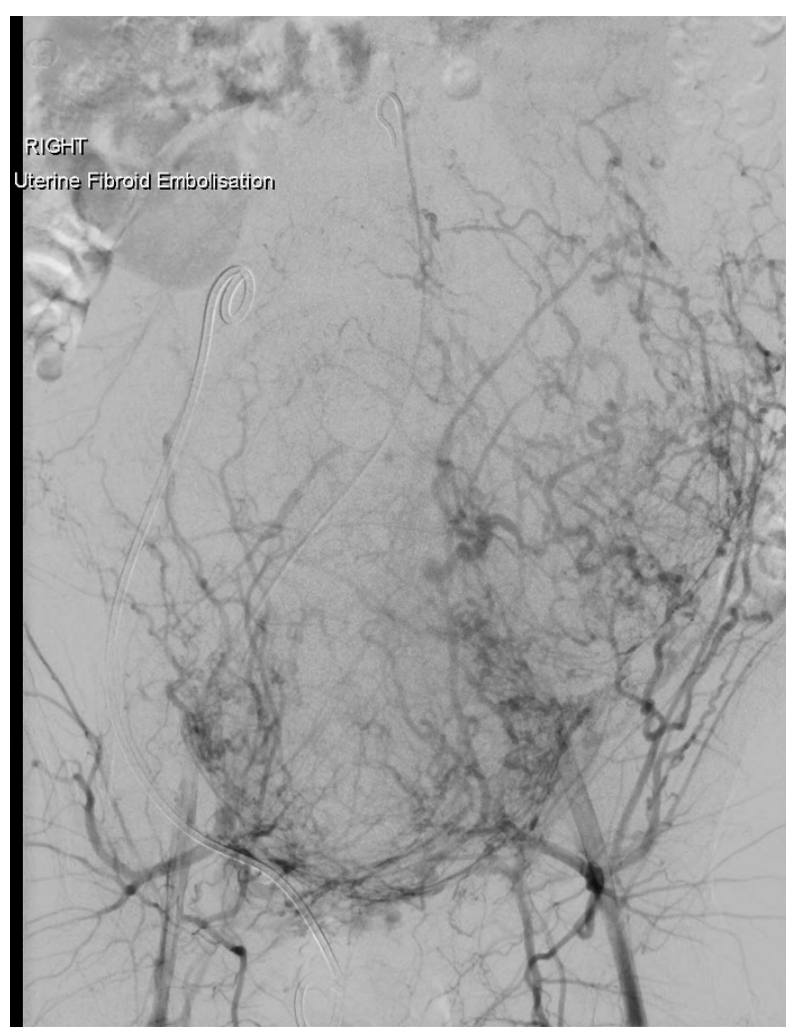

Figure 2: Pre-operative fluoroscopy at time of fibroid embolisation in a patient with Psuedo-Meigs Syndrome.

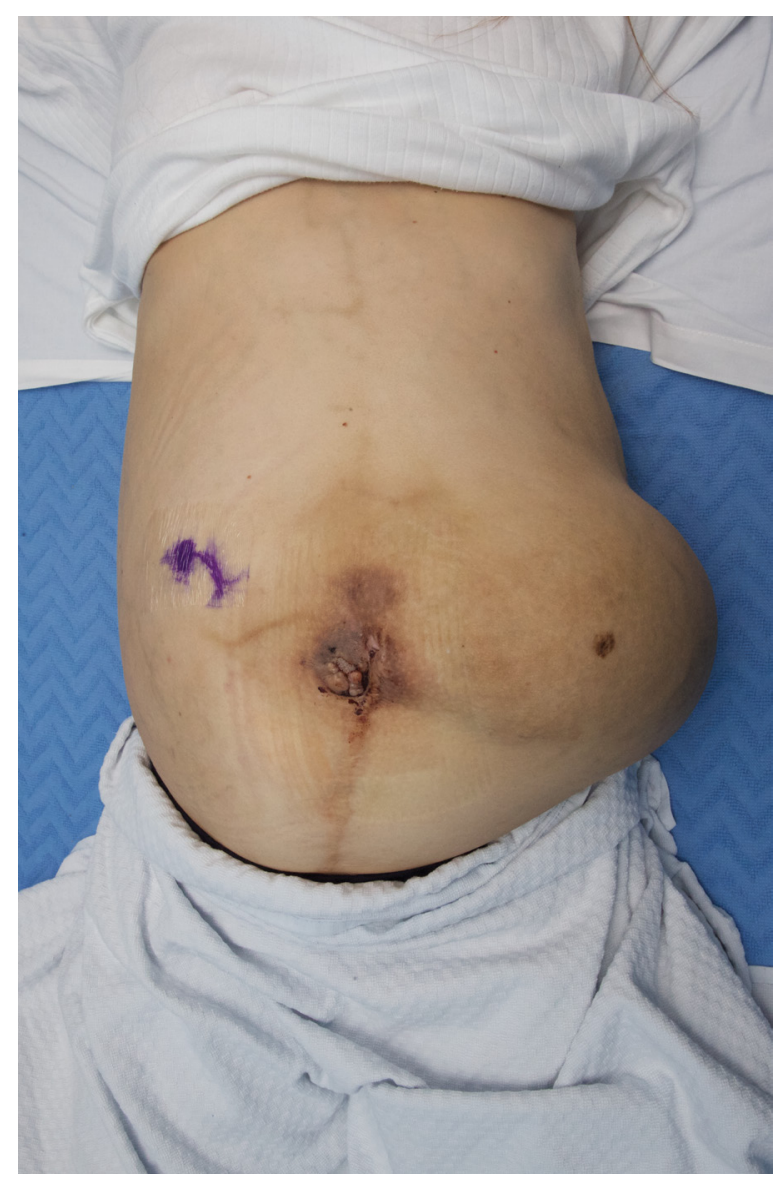

Figure 3: Pre-operative examination of a patient with Psuedo-Meigs Syndrome.

or colon cancers where there is presence of peritoneal metastasis.

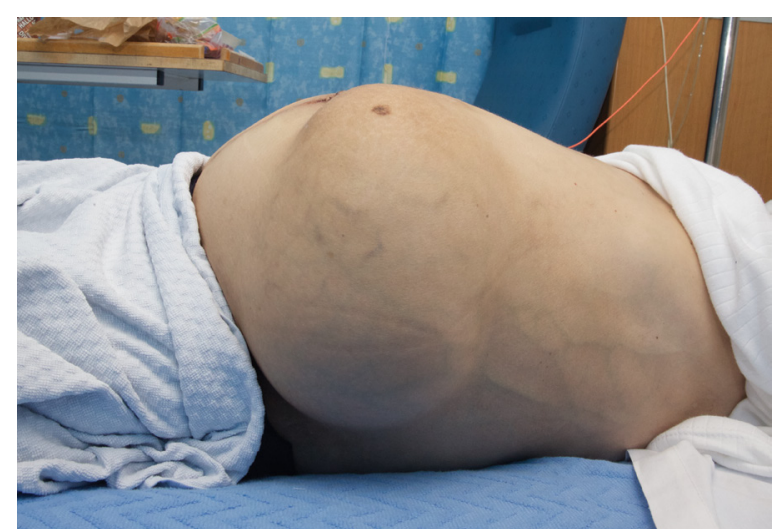

Figure 4: Pre-operative clinical examination (lateral view) of a patient with Psuedo-Meigs Syndrome.

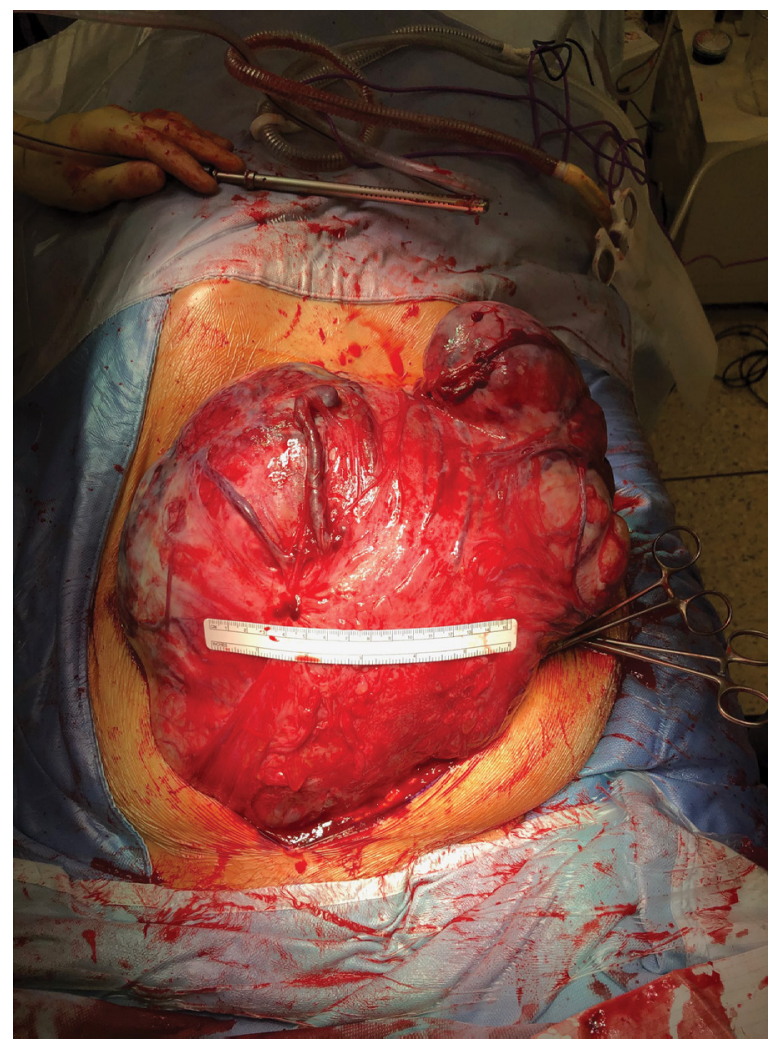

Figure 5: Intra-operative Pelvic mass.

There have been previous reported cases of Pseudo Meigs syndrome secondary to uterine leiomyomas with reported elevated Ca 125 ranging between 83-1854 $[6,9]$. There have been previous case reports of ascites + - pleural effusion in the setting of endometriosis. In those cases the ascitic or pleuritic fluid was frequently reported as blood stained with the haemosiderin content making interpretation of fluid cytology challenging [10]. Our reported case is unique and is in contrast to the prior reports of pseudo Meigs syndrome describing multiple pathologies of leiomyoma, adenomyosis and extensive endometriosis. In addition, our presented case highlights that in the setting of benign fluid cytology and benign pelvic biopsy that fertility conserving surgery is a reasonable alternative (Figure 1, Figure 2, Figure 3, Figure 4, Figure 5, Figure 6, Figure 7, Figure 8, 


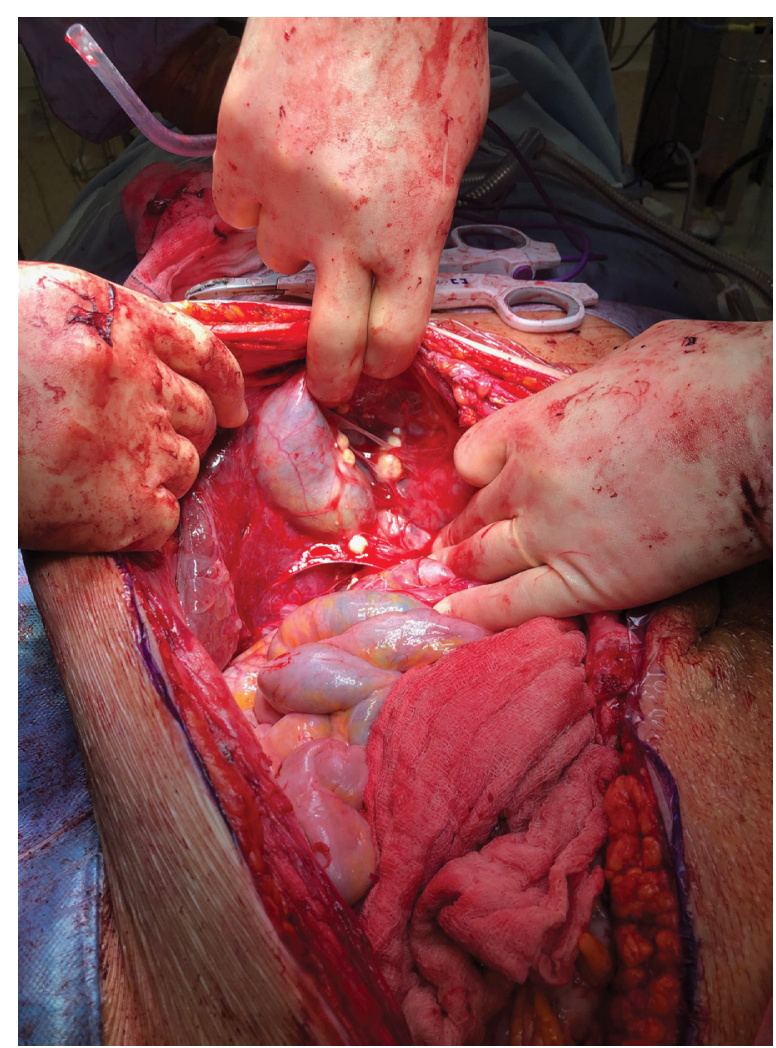

Figure 6: Intra-operative peri-hepatic calcified lesions.

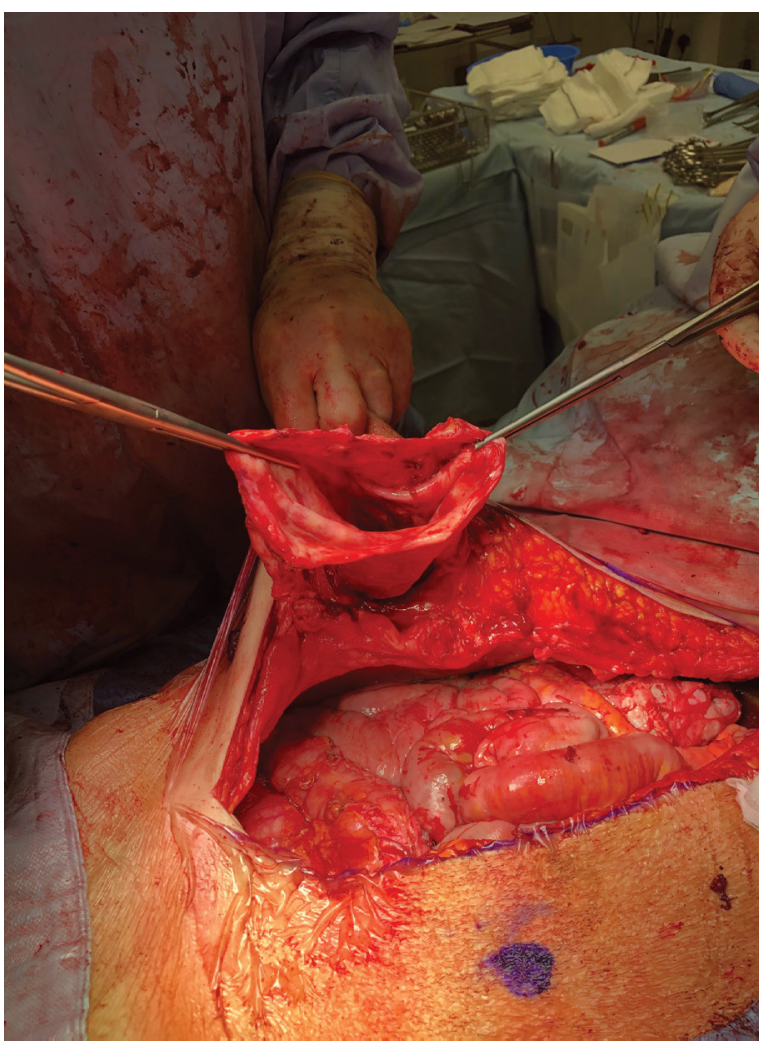

Figure 7: Left abdominal wall subcuticular seroma/ Endometriotic cyst capsule.

Figure 9 and Figure 10).

\section{Funding}

No funding sources for this submission.

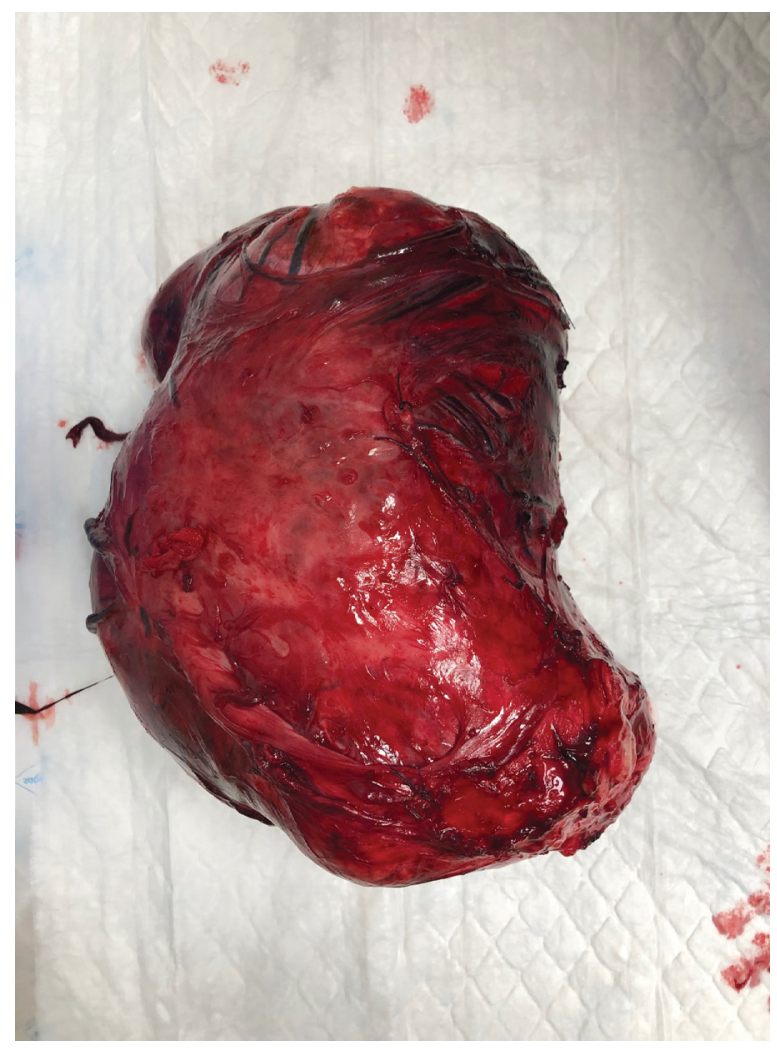

Figure 8: Excised pelvic mass Anterior view.

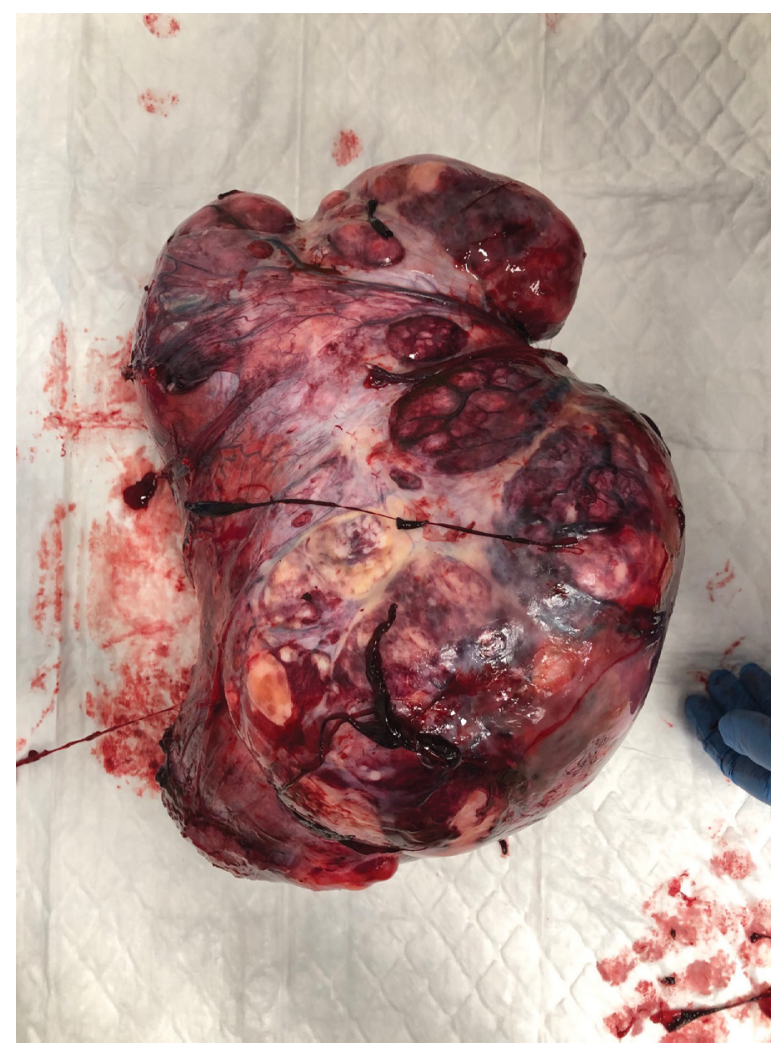

Figure 9: Excised pelvic mass Posterior view.

\section{Authors Declaration}

All authors have had equal contribution to this submission and all authors report no conflicts of interest. 


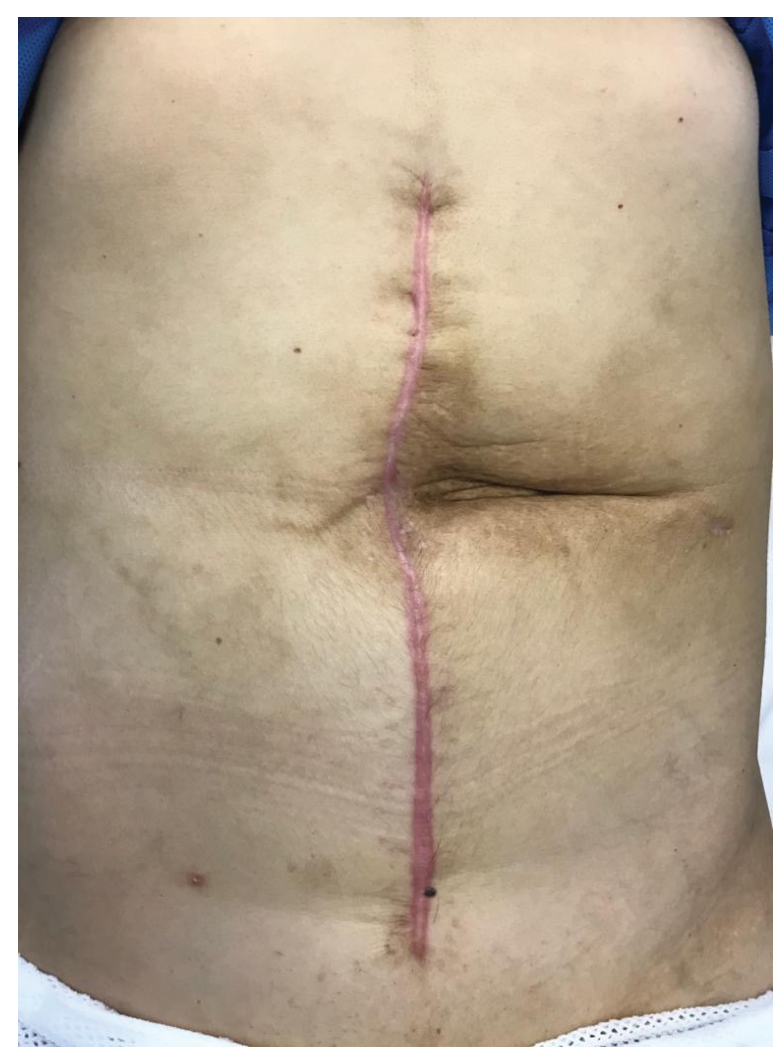

Figure 10: Three month post-operative abdominal examination of a patient with Psuedo-Meigs Syndrome.

\section{References}

1. Gaughan EMG, Walsh TA (2014) Risk-reducing surgery for women at high risk of epithelial ovarian cancer. The Obstetrician \& Gynaecologist 16: 185-191.

2. Berek JS, Kehoe ST, Kumar L, Friedlander M (2018) Cancer of the ovary, fallopian tube, and peritoneum. Int J Gynaecol Obstet 143: 59-78.

3. Meigs JV (1954) Fibroma of the ovary with ascites and hydrothorax; Meigs' syndrome. Am J Obstet Gynecol 67: 962-985.

4. Peparini N, Chirletti $P$ (2009) Ovarian malignancies with cytologically negative pleural and peritoneal effusions: Demons' or meigs' pseudo-syndromes? Int J Surg Pathol 17: 396-397.

5. Younas K, Hadoura E, Majoko F, Bunkheila A (2016) A review of evidence-based management of uterine fibroids. The Obstetrician \& Gynaecologist 18: 33-42.

6. Dong RY, Jin CJ, Zhang Q, Yang XS, Kong BH (2015) Cellular leiomyoma with necrosis and mucinous degeneration presenting as pseudo-Meigs' syndrome with elevated CA125. Oncol Rep 33: 3033-3037.

7. Fremed MA, Levin TL, Sun KY, Weiser DA (2015) Ovary preservation in the treatment of childhood Meigs syndrome. Pediatr Blood Cancer 62: 2011-2014.

8. Bast RC, Klug TL, St John E, Jenison E, Niloff JM, et al. (1983) A radioimmunoassay using a monoclonal antibody to monitor the course of epithelial ovarian cancer. $\mathrm{N}$ Engl J Med 309: 883-887.

9. Adlan A-S, Chua PT, Huang K-G (2010) Laparoscopic resection of a subserosal leiomyoma presenting as pseudo-meigs' syndrome. Journal of Gynecologic Surgery 36: 222-225.

10. Gungor T, Kanat-Pektas M, Ozat M, Zayifoglu Karaca M (2011) A systematic review: Endometriosis presenting with ascites. Arch Gynecol Obstet 283: 513-518. 